\title{
Effect of Periodontal Infection on Birth Weight of Infants: A Clinical and Microbiological Study*
}

\author{
Shaila Kothiwale ${ }^{\#}$, Megha Gandhi \\ Department of Periodontics, KLE V. K. Institute of Dental Sciences, KLE University, Belgaum, India \\ Email: " shailakothiwale2000@yahoo.co.in
}

Received September 10, 2012; revised October 10, 2012; accepted October 18, 2012

Copyright (C) 2013 Shaila Kothiwale, Megha Gandhi. This is an open access article distributed under the Creative Commons Attribution License, which permits unrestricted use, distribution, and reproduction in any medium, provided the original work is properly cited.

\begin{abstract}
Background: Maternal periodontal infection has been proposed to influence pre-term delivery and low birth weight infants through mechanisms involving inflammatory mediators or direct bacterial assault on the amnion. Thus, the aim of the present study was to assess the presence of periodontal pathogens in maternal periodontal infection and their effect on the birth weight of infants. Materials and Methods: The case-control study included 30 mothers with a singleton gestation and gestational age of $>37$ weeks. Data regarding the periodontal status, pregnancy outcome variables, hemoglobin levels $(\mathrm{Hb} \%)$ and other factors that may influence adverse pregnancy outcomes were collected. A qualitative analysis of the predominant anaerobic organisms was conducted. The data was analysed using one-way ANOVA test and Scheffe's test. Results: The microbiological results showed that prevalent colonies of organisms isolated from the experimental groups were of Bacteroides spp. $(\mathrm{n}=3)$, Fusobacterium spp. $(\mathrm{n}=3)$ and Peptostreptococcus spp. $(\mathrm{n}=$ 9). In the control group, Peptostreptococcus spp. $(\mathrm{n}=15)$ were predominant. In the experimental group, it was seen that Bacteroides spp. $(p=0.003)$ and Fusobacterium spp. $(p=0.050)$ were statistically significant with the birth weight of the infant. The one-way ANOVA test showed that the birth weight of the infant was inversely proportionate with increase in severity of the periodontal disease. Scheffe's test showed that presence of a periodontal pocket showed most significance to the birth weight of an infant as compared to presence of local factors or bleeding on probing. Conclusion: The study revealed that the gram negative organisms, Bacteroides spp. and Fusobacterium spp. were significantly associated with the low birth weight (LBW) of the infants, although there was not a significant difference in the clinical periodontal status between the experimental groups and the controls. It could be proposed that specific groups of pathogens may have a contributory role in the risk of LBW.
\end{abstract}

Keywords: Periodontal Infection; Bacteroides; Fusobacterium; Gram Negative Organisms; Low Birth Weight

\section{Introduction}

According to UNICEF, every year, about 18 million infants are born with low birth weight globally [1]. A Human Development Report UNDP in the year 2005 reported that the incidence of low birth weight (LBW) in India was $30 \%$, being the second leading country among its member countries [2]. The Government of India, along with the strategies developed to achieve "Health for All" by the year 2000, wished to decrease incidence of LBW to $10 \%$. Incidence of LBW in India in the year 2008 was $30 \%$. This target has not been achieved till today; in fact we are far away from the target [3]. The international definition of low birth weight for infants

${ }^{*}$ Conflict of interest: None.

${ }^{\#}$ Corresponding author. adopted by the Twenty-ninth World Health Assembly in 1976 is a birth weight of "less than 2500 g" (up to and including $2499 \mathrm{~g}$ ) [4]. LBW infants carry relatively higher risk of perinatal and neonatal mortality and substandard growth and development subsequently.

The various consequences of LBW are long-term disability such as congenital anomalies, chronic respiratory diseases and neurodevelopmental disabilities like cerebral palsy, poor growth, increased incidence of adult diseases, type 2 diabetes mellitus, hypertension, cardiovascular diseases and an additional risk for girls is having smaller babies when they become mothers [5-9].

There are various risk factors for LBW like high ( $>34$ years) and low ( $<17$ years), maternal age, race, genetic background, low socio-economic status, any adverse habits - drug, alcohol and tobacco use, multiple pregnan- 
cies, maternal stress, hypertension, genitourinary tract infections, inadequate prenatal care, poor oral hygiene, short gestational age. However, these risk factors are present in approximately one fourth of preterm LBW experimental groups, leading to a continued search for other causes [6].

Periodontal diseases are a group of infectious diseases caused by predominantly gram-negative, anaerobic, and microaerophilic bacteria that colonise the subgingival area and cause local and systemic elevations of pro-inflammatory prostaglandins and cytokines [10]. A few decades ago, it was hypothesised that maternal periodontal infection might be related to LBW [11]. A study showed that pregnant hamsters subcutaneously infected with Porphyromonas gingivalis tended to produce low birth weight pups [12]. Maternal periodontal infection has been proposed to influence pre-term delivery and low birth weight infants through mechanisms involving inflammatory mediators or direct bacterial assault on the amnion. Thus, the aim of the present study was to assess the presence of periodontal pathogens in maternal periodontal infection and their effect on the birth weight of infants.

\section{Materials \& Methods}

The study was a case-control study conducted at the free labour ward, KLE's Dr. Prabhakar Kore Hospital \& Research Centre at Belgaum. Mothers within the age group of 18 - 35 years with a singleton gestation and gestational age of $>37$ weeks were included in the study. They were divided equally into experimental groups and controlsexperimental groups including mothers delivering infants with a low birth weight $(<2500 \mathrm{~g})$ and controls including mothers delivering infants with a normal birth weight $(>2500 \mathrm{~g})$. Mothers with systemic genitourinary tract infections, multiple pregnancies, pre eclampsia or eclampsia and gestational diabetes and conditions like diabetes, cardiovascular disorders, hypertension, and severe anemia were excluded from the study. Ethical clearance was obtained from the institutional committee.

An informed consent was obtained from all subjects before inclusion in the study. Data was collected from hospital case records as well as direct interaction with the subjects (about age, hemoglobin levels, obstetric history, history of dental treatment, antenatal care, gestational age and sex and birth weight of the newborn). Periodontal status was assessed using Table 1.

For microbiological analysis, subgingival plaque samples were collected from all quadrants in the oral cavity and pooled together. The sites, with deepest pocket depth in each quadrant, were identified and isolated with cotton rolls. A sterile curette or a scaler was used to collect the plaque samples which were then immediately transported to the laboratory in thioglycollate broth for microbiological analysis. One loopful of sample was then inocu-
Table 1. Community periodontal index.

\begin{tabular}{cl}
\hline Score & Criteria \\
\hline 0 & Healthy. \\
1 & $\begin{array}{l}\text { Bleeding observed, directly or using a mouth mirror, after } \\
\text { probing. }\end{array}$ \\
2 & $\begin{array}{l}\text { Calculus detected during probing, but all of the black band } \\
\text { on the probe visible. }\end{array}$ \\
3 & $\begin{array}{l}\text { Pocket } 4-5 \mathrm{~mm} \text { (gingival margin within the black band } \\
\text { on the probe). }\end{array}$ \\
4 & Pocket 6 mm or more (black band on the probe not visible). \\
$\mathrm{X}$ & Excluded sextant (less than two teeth present). \\
9 & Not recorded.
\end{tabular}

lated onto blood agar supplemented with Haemin (5 $\mu \mathrm{g} / \mathrm{ml})$ and Vitamin $\mathrm{K}(10 \mu \mathrm{g} / \mathrm{ml})$. Blood agar plates used for anaerobic isolation were prepared with Brucella agar base. Plates were incubated in anaerobic conditions for 3 - 5 days at $37^{\circ} \mathrm{C}$. The method used for obtaining anaerobiosis in the jar was "Internal Gas Generating system". After 72 hours of incubation at $37^{\circ} \mathrm{C}$, the anaerobic jar was opened. The plates were examined for the presence of colonies. When the colonies appeared on the anaerobic plates, the microorganisms were then identified under the microscope.

Data were entered and analysed using a statistical program, MedCalc 10.2 software. The level of significance was 0.05. One-way ANOVA test was done to assess the mean values of the measured parameters followed by the Scheffe's test for pair-wise comparisons.

\section{Results}

The demographic status of the study population is presented in Figure 1. The parameters (age, hemoglobin levels, gestational age) between experimental groups and controls were statistically non-significant. The study population was homogeneous in nature with respect to the socioeconomic status and literacy levels of the mothers. The mothers had no adverse oral habits except one mother who had a history of betel nut chewing. Among all the mothers, only one had not received the antenatal care.

The microbiological results showed that prevalent colonies of organisms isolated from the experimental groups were of Bacteroides spp. $(\mathrm{n}=3)$, Fusobacterium spp. $(\mathrm{n}=3)$ and Peptostreptococcus spp. $(\mathrm{n}=9)$. Peptostreptococcus spp. $(\mathrm{n}=15)$ were predominant in the control group (Figure 2).

Further correlating the presence of these bacteria with the periodontal status among Experimental groups, it showed that the gram negative anaerobes, Bacteroides spp. and Fusobacterium spp., were present in subjects with CPI scores of 3 i.e. presence of pockets $<5.5 \mathrm{~mm}$, while Peptostreptococcus spp., a gram positive anaerobe was present in increased prevalence in subjects with a 


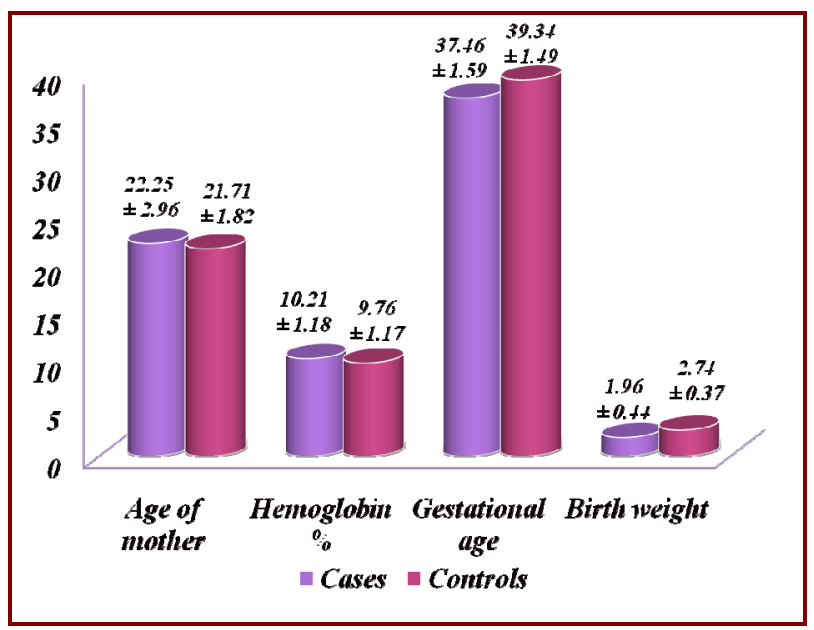

Figure 1. Demographic status of the study population.

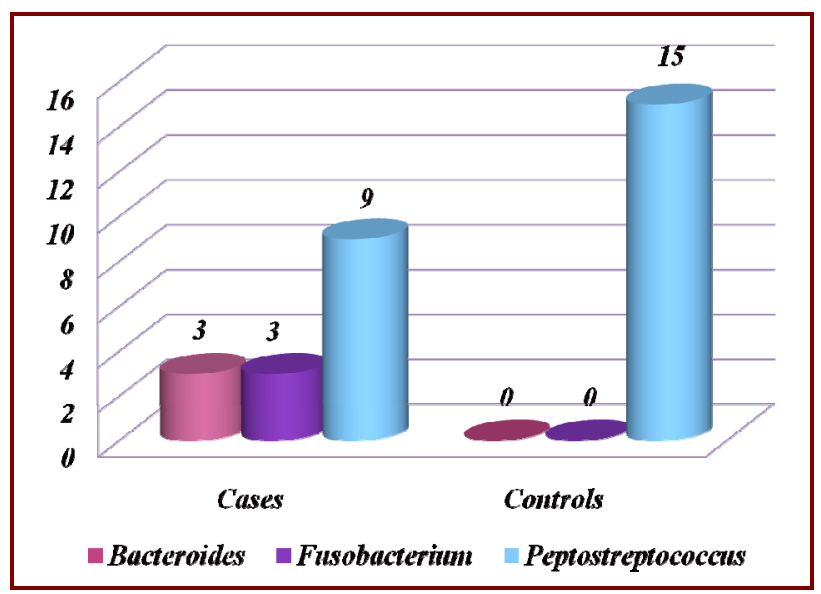

Figure 2. Presence of microorganisms in experimental groups and Controls.

CPI score of 2 i.e. presence of plaque and calculus (Figure 3). The Bacteroides spp. and Fusobacterium spp. were associated with periodontal disease, while Peptostreptococcus spp. was prevalent in mothers with lower CPI scores delivering low birth weight infants or those delivering normal birth weight infants.

When the three organisms were correlated with the birth weight of the infant among the experimental groups, the one-way ANOVA test showed high significance $(\mathrm{p}=$ 0.002 ) which meant that the experimental groups were non-homogenous (Table 2). Thus, Scheffe's test was carried out to study the correlation of each of the organisms. It was seen that Bacteroides spp. $(\mathrm{p}=0.003)$ and Fusobacterium spp. $(\mathrm{p}=0.050)$ were statistically significant with the birth weight of the infant as compared to Peptostreptococcus spp. (Table 3).

When the periodontal status was correlated with the birth weight of the infant among the controls, the oneway ANOVA test showed no significance $(p=0.150)$ (Table 4).

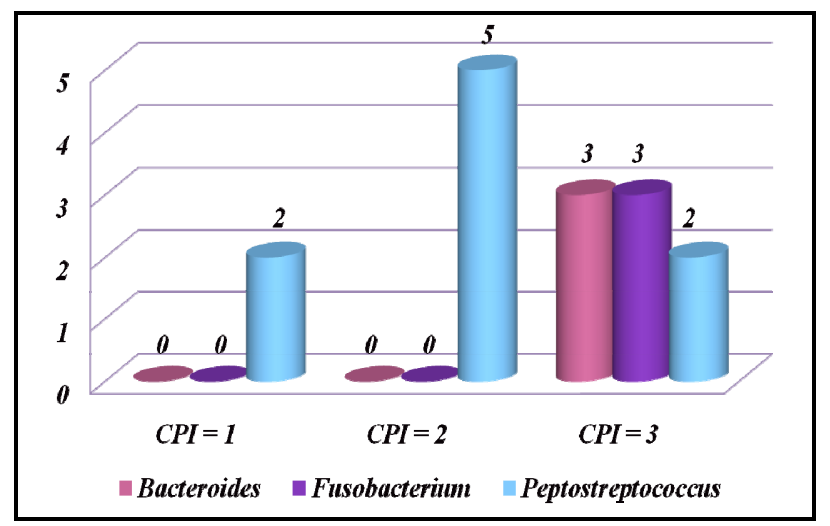

Figure 3. Correlation of microorganisms with periodontal status among experimental groups.

Table 2. Correlation of microorganisms with birth weight of infant among experimental groups using one-way ANOVA test.

\begin{tabular}{cccccc}
\hline & $\begin{array}{c}\text { Sum of } \\
\text { squares }\end{array}$ & df & $\begin{array}{c}\text { Mean } \\
\text { square }\end{array}$ & F & p value \\
\hline Between groups & 1.770 & 2 & 0.885 & 10.766 & $0.002^{*}$ \\
Within groups & 1.069 & 13 & 0.082 & & \\
Total & 2.839 & 15 & & & \\
\hline
\end{tabular}

*Statistically significant.

Table 3. Correlation of microorganisms with birth weight of infant among experimental groups using Scheffe's test.

\begin{tabular}{lcc}
\hline & Mean difference & p value \\
\hline Bacteroides: peptostreptococcus & $-0.80500^{*}$ & $0.003^{*}$ \\
Fusobacterium: peptostreptococcus & $-0.52167^{*}$ & $0.050^{*}$ \\
Bacteroides: fusobacterium & -0.28333 & 0.500 \\
\hline
\end{tabular}

*Statistically significant.

Table 4. Correlation of periodontal status with birth weight of infant among controls using one-way ANOVA test.

\begin{tabular}{cccccc}
\hline & $\begin{array}{c}\text { Sum of } \\
\text { squares }\end{array}$ & df & $\begin{array}{c}\text { Mean } \\
\text { square }\end{array}$ & F & p value \\
\hline Between groups & 1.023 & 2 & 0.511 & 2.204 & $0.150^{*}$ \\
Within groups & 3.016 & 13 & 0.232 & & \\
Total & 4.038 & 15 & & & \\
\hline
\end{tabular}

*Statistically non-significant.

When the periodontal status was correlated with the birth weight of the infant among the experimental groups, the one-way ANOVA test showed that the birth weight of the infant was inversely proportionate with increase in severity of the periodontal disease $(p=0.008)$ (Table 5). On further conducting Scheffe's test, it was observed that presence of a periodontal pocket with bleeding on probing and calculus showed most significance to the birth weight of an infant as compared to presence of local factors or bleeding on probing (Table 6). 
Table 5. Correlation of periodontal Status with birth weight of infant among experimental groups using one-way ANOVA test.

\begin{tabular}{cccccc}
\hline & $\begin{array}{c}\text { Sum of } \\
\text { squares }\end{array}$ & df & $\begin{array}{c}\text { Mean } \\
\text { square }\end{array}$ & F & p value \\
\hline Between groups & 1.478 & 2 & 0.739 & 7.053 & $0.008^{*}$ \\
Within groups & 1.362 & 13 & 0.105 & & \\
Total & 2.839 & 15 & & & \\
\hline
\end{tabular}

*Statistically significant.

Table 6. Correlation of periodontal Status with birth weight of infant among experimental groups using Scheffe's test.

\begin{tabular}{lcc}
\hline & $\begin{array}{c}\text { Mean } \\
\text { difference }\end{array}$ & p value \\
\hline Presence of pocket: bleeding on probing & 0.71875 & $0.046^{*}$ \\
Presence of pocket: presence of calculus & 0.56042 & $0.023^{*}$ \\
Bleeding on probing: presence of calculus & 0.15833 & 0.838 \\
\hline
\end{tabular}

*Statistically significant.

\section{Discussion}

The case-control study was conducted to test the hypothesis that "maternal periodontal infection might create a higher risk for LBW of infants". The subgingival plaque samples were collected from all quadrants of the oral cavity to better discriminate the likelihood of accumulation of any specific bacterial species.

The data analysed revealed that the samples were homogenous for the different maternal variables including maternal age, maternal hemoglobin levels, obstetric history, literacy, socioeconomic status, antenatal care and the gestational age (Figure 1). Only one mother had not received complete antenatal care but this did not influence the results of the study. All the mothers, except one, included in the study did not have any adverse oral habits. Mitchell-Lewis et al. (2001) suggested that a study of the impact of infection by specific periodontal microbiota on pregnancy outcomes should involve only women with undisturbed subgingival plaques during pregnancy [13]. Accordingly, the study population consisted of women enrolled immediately after delivery and almost none of these women had visited a dentist during their pregnancy periods.

Thus, the study was free of selection biases as all the cofounding variables which may affect the birth weight of the infant and the maternal periodontal disease were controlled for.

Microbiologic analyses of plaque samples from the mothers revealed the presence of Gram positive organisms i.e. Peptostreptococcus spp., as well as gram negative organisms i.e. Bacteroides spp. and Fusobacterium spp., associated with periodontal disease. Controls showed the presence of Peptostreptococcus spp. while the experimental groups showed a greater presence of Bacteroides spp. and Fusobacterium spp.

Minkoff et al. (1984) studied 233 women with preterm rupture of membrane (PROM) [14]. They found women colonised with Bacteroides spp. had a significantly more frequent preterm rupture of membrane, preterm delivery or a smaller infant (LBW infant). Bacteroides spp. are known to release various protease enzymes which degrade different tissue components like collagen, proteoglycans, etc. Collagen is a major component of the amniotic membrane and plays a large role in the stress tolerance of the fetal membrane, thus protecting against the occurrence of premature rupture of membrane. Bacteroides spp. have been observed to reduce the chorioamnion membrane strength in vitro due to its protease-releasing property [15].

Periodontopathogens may also contribute to the development of LBW infants, either by direct contamination of the fetoplacental unit, or their endotoxins like LPS may infect the fetoplacental unit through bacteremia. Katz et al. (2009) observed that gram negative periodontopathogens get localised in the placenta resulting in chorioamnionitis, infected amniotic fluid or a fetal inflammatory response [16]. This has an adverse outcome on the gestational age as well as fetal growth. The lipopolysaccharides released by the pathogens may also initiate an inflammatory response which evokes a maternal as well as a fetal response, resulting in LBW of the infant $[6,8,17]$. K. Hasegawa-Nakamura et al. (2011) identified gram negative periodontopathogens in the chorionic tissues of hospitalised high-risk pregnant women and subsequently investigated that the LPS released from these organisms provoke an inflammatory response [18].

A study conducted by Buduneli N. et al. (2005) showed results of high levels of $P$. micros and $C$. rectus in mothers delivering LBW infants [19]. A study from a cohort of young minority women in New York, have found that PLBW mothers had significantly higher levels of $T$. forsythensis and C. rectus, although clinical periodontal status revealed no significant differences between the two groups [13]. It could be suggested that the possible relationship between periodontal disease and LBW, reported previously, may probably be explained by complex actions of different periodontal bacteria in the subgingival plaque. It is also likely that more than the clinical periodontal indices, it could be the prevalence of subgingival pathogens which may increase the circulating levels of cytokines or prostaglandins, in turn increasing the risk for LBW.

In conclusion, the study revealed that the gram negative organisms, Bacteroides spp. and Fusobacterium spp. were significantly associated with the low birth weight of the infants, although there was not a significant differ- 
ence in the clinical periodontal status between the experimental groups and the controls. The difference in the distribution of specific periodontal pathogens and their effect on the fetoplacental unit to regulate the host immune and inflammatory response in a pregnant mother may give rise to decreased fetal weight. It could be proposed that maternal periodontal infection may have a contributory role in the risk of LBW.

\section{Acknowledgements}

Dr. Jyoti Nagmoti M.D., Ph.D., Department of Microbiology, Jawaharlal Nehru Medical College, KLE University.

\section{REFERENCES}

[1] UNICEF \& WHO, "Low Birthweight: Country, Regional and Global Estimates," UNICEF, 2004.

[2] United Nations Development Programme Human Development Report, 2005.

[3] M. K. Sharma, D. Kumar, A. Huria and P. Gupta, "Maternal Risk Factors of Low Birth Weight in Chandigarh India," The Internet Journal of Health, Vol. 9, No. 1, 2009.

[4] World Health Organization, "The Incidence of Low Birth Weight: An Update," Weekly Epidemiological Record, Vol. 59, 1984, pp. 205-211.

[5] S. Offenbacher, V. Katz, G. Fertik, J. Collins, D. Boyd and G. Maynor, "Periodontal Infection as a Possible Risk Factor for Preterm Low Birth Weight," Journal of Periodontology, Vol. 67, No. 10s, 1996, pp. 1103-1113. doi:10.1902/jop.1996.67.10s.1103

[6] S. Offenbacher, H. L. Jared, P. G. O'Reilly, et al., "Potential Pathogenic Mechanisms of Periodontitis Associated Pregnancy Complications," Annals of Periodontology, Vol. 3, No. 1, 1998, pp. 233-250.

doi:10.1902/annals.1998.3.1.233

[7] C. M. Champagne, P. N. Madianos, S. Lieff, et al., "Periodontal Medicine: Emerging Concepts in Pregnancy Outcomes," Journal of the International Academy of Periodontology, Vol. 2, No. 1, 2000, pp. 9-13.

[8] F. A. Scannapieco, R. B. Bush and S. Paju, "Periodontal Disease as a Risk Factor for Adverse Pregnancy Outcomes. A Systematic Review," Annals of Periodontology, Vol. 8, No. 1, 2003, pp. 70-78. doi:10.1902/annals.2003.8.1.70

[9] X. Xiong, P. Buekens, W. D. Fraser, J. Beck and S. Offenbacher, "Periodontal Disease and Adverse Pregnancy Outcomes: A Systematic Review," BJOG: An International
Journal of Obstetrics \& Gynaecology, Vol. 113, No. 2, 2006, pp. 135-143. doi:10.1111/j.1471-0528.2005.00827.x

[10] R. C. Page and K. S. Kornman, "The Pathogenesis of Human Periodontitis: An Introduction," Periodontology, Vol. 14, No. 1, 2000, pp. 9-11. doi:10.1111/j.1600-0757.1997.tb00189.x

[11] J. A. McGregor, J. I. French, R. Richter, et al., "Antenatal Microbiologic and Maternal Risk Factors Associated with Prematurity," American Journal of Obstetrics \& Gynecology, Vol. 163, No. 5, 1990, pp. 1465-1473. doi:10.1016/0002-9378(90)90607-9

[12] J. G. Collins, M. A. Smith, R. R. Arnold and S. Offenbacher, "Effects of Escherichia coli and Porphyromonas gingivalis Lipopolysaccharides on Pregnancy Outcome in the Golden Hamster," Infection and Immunity, Vol. 62, No. 10, 1994, pp. 4652-4655.

[13] D. Mitchell-Lewis, S. P. Engebretson, J. Chen, I. B. Lamster and P. N. Papapanou, "Periodontal Infections and Pre-Term Birth: Early Findings from a Cohort of Young Minority Women in New York," European Journal of Oral Sciences, Vol. 109, No. 1, 2001, pp. 34-39. doi:10.1034/j.1600-0722.2001.00966.x

[14] H. Minkoff, A. N. Grunebaum, R. H. Schwarz, et al., "Risk Factors for Prematurity and Premature Rupture of Membranes: A Prospective Study of the Vaginal Flora in Pregnancy," American Journal of Obstetrics \& Gynecology, Vol. 150, No. 8, 1984, pp. 965-972.

[15] J. A. McGregor, J. I. French, D. Lawellin, et al., "Bacterial Protease-Induced Reduction of Chorioamniotic Membrane Strength and Elasticity," Obstetrics \& Gynecology, Vol. 69, No. 2, 1987, pp. 167-174.

[16] J. Katz, N. Chegini, K. T. Shiverick and R. J. Lamont, "Localization of $P$. gingivalis in Preterm Delivery Placenta," Journal of Dental Research, Vol. 88, No. 6, 2009, pp. 575-578. doi:10.1177/0022034509338032

[17] M. K. Jeffcoat, N. C. Geurs, M. S. Reddy, et al., "Periodontal Infections and Preterm Birth: Results of a Prospective Study," Journal of Dental Research, Vol. 80, 2001, p. 956.

[18] K. Hasegawa-Nakamura, F. Tateishi, T. Nakamura, et al., "The Possible Mechanism of Preterm Birth Associated with Periodontopathic Porphyromonas gingivalis," Journal of Periodontal Research, Vol. 46, No. 4, 2011, pp. 497-504. doi:10.1111/j.1600-0765.2011.01366.x

[19] N. Buduneli, H. Baylas, E. Buduneli, et al., "Periodontal Infections and Pre-Term Low Birth Weight: A CaseControl Study," Journal of Clinical Periodontology, Vol. 32, No. 2, 2005, pp. 174-181. doi:10.1111/j.1600-051X.2005.00670.x 